\title{
Hyperbolic Asymptotics in Burgers' Turbulence and Extremal Processes
}

\author{
S.A. Molchanov ${ }^{1}$, D. Surgailis ${ }^{2}$, W.A. Woyczynski ${ }^{3}$ \\ 1 Department of Mathematics, University of Southern California, Los Angeles, CA, USA \\ 2 Institute of Mathematics and Cybernetics, Lithuanian Academy of Sciences, Vilnius, Lithuania \\ 3 Center for Stochastic and Chaotic Processes in Science and Technology, Case Western Reserve \\ University, Cleveland, Ohio 44106, USA
}

Received: 28 March 1994

Abstract: Large time asymptotics of statistical solution $u(t, x)(1.2)$ of the Burgers' equation (1.1) is considered, where $\xi(x)=\xi_{L}(x)$ is a stationary zero mean Gaussian process depending on a large parameter $L>0$ so that

$$
\xi_{L}(x) \sim \sigma_{L} \eta(x / L) \quad(L \rightarrow \infty),
$$

where $\sigma_{L}=L^{2}(2 \log L)^{1 / 2}$ and $\eta(x)$ is a given standardized stationary Gaussian process. We prove that as $L \rightarrow \infty$ the hyperbolicly scaled random fields $u\left(L^{2} t, L^{2} x\right)$ converge in distribution to a random field with "saw-tooth" trajectories, defined by means of a Poisson process on the plane related to high fluctuations of $\xi(x)$, which corresponds to the zero viscosity solutions. At the physical level of rigor, such asymptotics was considered before by Gurbatov, Malakhov and Saichev (1991).

\section{Introduction}

The Burgers' equation

$$
\partial_{t} u+u \partial_{x} u=\mu \partial_{x}^{2} u
$$

$t>0, x \in \mathbf{R}, u=u(t, x), u(0, x)=u_{0}(x)$, admits the well-known Hopf-Cole explicit solution

$$
u(t, x)=\frac{\int_{-\infty}^{\infty}[(x-y) / t] \exp \left[(2 \mu)^{-1}\left(\xi(y)-(x-y)^{2} / 2 t\right)\right] d y}{\int_{-\infty}^{\infty} \exp \left[(2 \mu)^{-1}\left(\xi(y)-(x-y)^{2} / 2 t\right)\right] d y}
$$

where $\xi(x)=-\int_{-\infty}^{x} u_{0}(y) d y$ (see Hopf (1950)). It describes propagation of nonlinear hyperbolic waves, and has been considered as a model equation for various physical phenomena from the hydrodynamic turbulence (see e.g. Chorin (1975)) to evolution of the density of matter in the Universe (see Shandarin, Zeldovich (1989)). Due to nonlinearity, solution (1.2) enters several different stages, including that of shock waves' formation, which are largely determined by the value of the Reynolds 
number $R=\sigma l / \mu$ (see Gurbatov, Malakhov, Saichev (1991)). Here, $\mu>0$ is the viscosity parameter, while $\sigma$ and $l$ have the physical meaning of characteristic scale and amplitude of $\xi(x)$, respectively.

Starting with Burgers' own papers (see Burgers' (1974) for an account of the early work in the area), numerous works discussed statistical solutions of (1.1), i.e., solutions corresponding to random initial data $\xi(x)=\xi(x ; \omega)$ (see, e.g., Kraichnan (1959)). The random process $\xi(x)$ is usually assumed to be stationary or having stationary increments. Although many of these works are not quite rigorous mathematically, they reflect the interest of physicists in the "Burgers' turbulence" and other physical phenomena described by this equation (for a survey of past and current work on the stochastic Burgers equation, see Fournier, Frisch (1983), Woyczynski (1993), Funaki, Surgailis, Woyczynski (1995), and other papers quoted in references).

From the probabilistic point of view, a study of the limiting behavior of $u(t, x)$ as $t \rightarrow \infty$, or as $\mu \rightarrow 0$, seems to be most interesting. If $\mu>0$ is fixed, then, under some additional (exponential) moment conditions on $\xi(x)$, and in absence of the long-range dependence, $u(t, x)$ obeys a "Gaussian scenario" of the central limit theorem type (see, e.g., Bulinskii, Molchanov (1991), Albeverio, Molchanov, Surgailis (1993)). Non-Gaussian limits have also been found under less restrictive conditions on $\xi$ (see e.g. Funaki, Surgailis, Woyczynski (1995)).

On the other hand, if the initial fluctuations $\xi(x)$ are large enough to make the exponential moments of $\xi(x)$ infinite, and the marginal tail distribution function

$$
P[\exp (\xi(x) / 2 \mu)>a]
$$

varies slowly as $a \rightarrow \infty$, then the behavior of $u(t, x)$ is very different from the "Gaussian scenario," namely,

$$
u(t, x) \sim \frac{x-y^{*}}{t} \quad(t \rightarrow \infty)
$$

where $y^{*}=y^{*}(t, x)$ is the point where $S(y):=\xi(y)-(x-y)^{2} / 2 t$ attains its maximum. For a degenerate shot noise process $\xi(x)$, the asymptotics $(1.3)$, together with an estimate of growth of the right-hand side of (1.3), was rigorously established in Albeverio, Molchanov, Surgailis (1995).

In their important physical works, Gurbatov, Malakhov, Saichev (1991) (see also Kraichnan (1968), and Fournier, Frisch (1983)) discussed asymptotics of $u(t, x)$ at high Reynolds numbers, in the case when the initial Gaussian data $\xi(x)$ are characterized by large "amplitude" $\sigma=\left(E(\xi(0))^{2}\right)^{1 / 2}$ and large "internal scale" $L=$ $\sigma / \sigma^{\prime}>>1$, where $\sigma^{\prime}=\left(E\left(\xi^{\prime}(0)\right)^{2}\right)^{1 / 2}$. At time $t=t_{L} \sim \ell_{L}\left(t_{L}\right)$, where

$$
\ell_{L}(t)=(\sigma t)^{1 / 2}\left(\log \left(\sigma^{\prime} t / 2 \pi L\right)\right)^{-1 / 4}
$$

is the "external scale" at time $t$, they demonstrated (at the physical level of rigor) that "[...] a strongly nonlinear regime of sawtooth waves [...] is set up, [...] and the field's statistical properties become self-preserving" (ibid., p. 163). In particular, they were able to find explicitly one- and two-point distribution functions of the (limit) sawtooth velocity process (ibid., Sect. 5.4).

In the present paper, we formulate the problem in mathematical terms and give a rigorous derivation of the "large internal scale asymptotics" of the above type in the sense of the weak convergence of finite dimensional distributions of hyperbolicly scaled velocity random field $u\left(L^{2} t, L^{2} x\right)$. The particular asymptotic form (2.1) of 
the initial Gaussian process is a simplification assumed for technical reasons; even in this case the proofs are rather involved. The limit "sawtooth" process, which corresponds to zero viscosity limit solutions of the Burgers' equation, is defined with the help of a Poisson process on $\mathbf{R}^{2}$ corresponding to high local maxima of the Gaussian data. The $n$-point distributions and correlation functions of the limit field are given. For $n=1,2$, they coincide with the corresponding expressions found by Gurbatov, Malakhov, Saichev (1991). The paper makes an extensive use of a modern theory of extremal processes; the comprehensive account thereof can be found in Leadbetter, Lindgren, Rootzen (1983).

In Sect. 2 we formally present our main result and take first steps towards its proof. Section 3 studies the Poisson convergence of high local maxima of the Gaussian processes together with the deterministic (parabolic) behavior of their trajectories near the extreme points. Section 4 introduces the Burgers $(\mathscr{B}-)$ topology on point processes - a natural topology for the problem at hand. The convergence and compactness criteria for that topology are then provided. In Sect. 5 we return to the study of the Hopf-Cole functional and complete the proof of our main Theorem 2.1. Finally, Sect. 6 discusses explicit formulas for the multipoint spacetime densities and correlation functions of the limit velocity field.

\section{Internal Scale and Hyperbolic Asymptotics}

The "internal scale" that was discussed above on the intuitive level will be formalized roughly as follows. We shall start with a zero-mean stationary differentiable Gaussian process $\eta(x)$ and take as the initial data process

$$
\xi_{L}(x)=\sigma_{L} \eta(x / L)
$$

where

$$
\sigma_{L}=L^{2} \sqrt{2 \log L} .
$$

The particular asymptotics of $\sigma_{L}$ is dictated by the standard normalization constant (see (2.7)) in the extremal theory of Gaussian processes, and the scaling properties of the Hopf-Cole functional (1.2). Then,

$$
\xi^{\prime}(x)=\left(\sigma_{L} / L\right) \eta^{\prime}(x / L)
$$

and the "internal scale"

$$
\frac{\left[E(\xi(x))^{2}\right]^{1 / 2}}{\left[E\left(\xi^{\prime}(x)\right)^{2}\right]^{1 / 2}}
$$

is proportional to parameter $L$. Studying the solutions at large "internal scales" will mean letting $L \rightarrow \infty$.

We shall assume that the covariance function $r(x)=E \eta(0) \eta(x)$ of the process $\eta(x), x \in \mathbf{R}$, satisfies the following two conditions:

$$
r(x)=o(1 / \log x) \quad(x \rightarrow \infty),
$$

and

$$
r(x)=1-\frac{1}{2 !} \lambda_{2} x^{2}+\frac{1}{4 !} \lambda_{4} x^{4}+o\left(x^{4}\right) \quad(x \rightarrow 0) .
$$




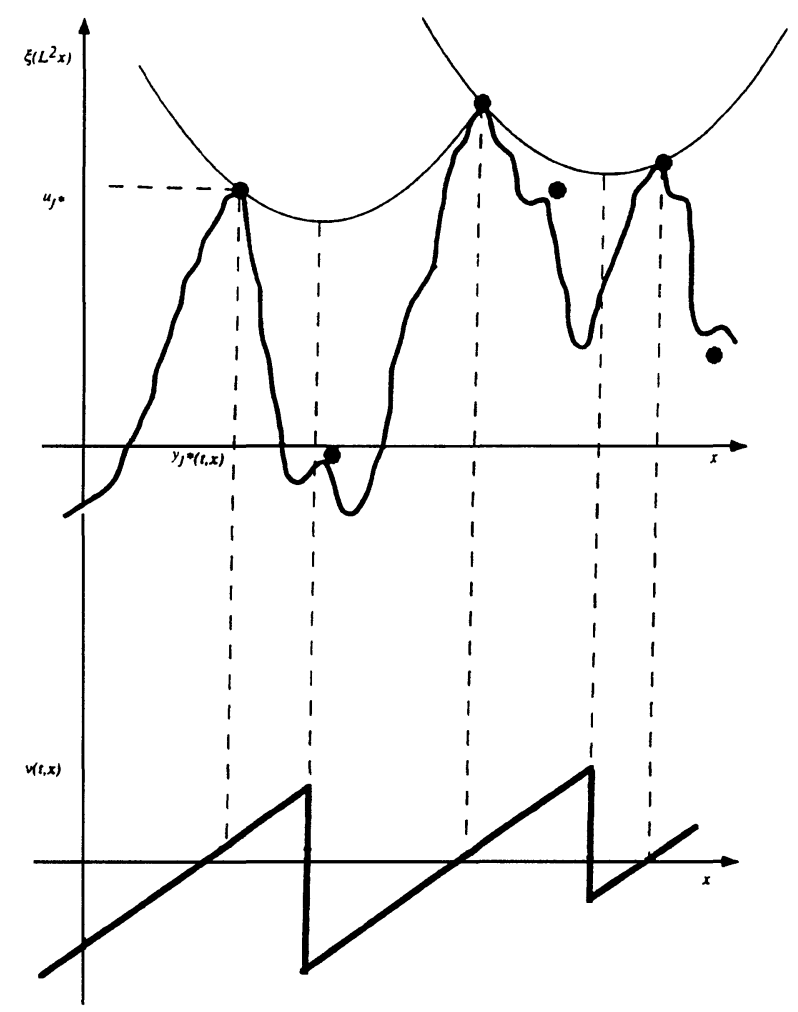

Fig. 1. Points $\left(y_{l}, u_{l}\right)$ of the Poisson process (marked by $\bullet$ ) correspond to high local maxima of the Gaussian curve $\xi(x)$. Critical parabolas define discontinuity points and zeros of the limit velocity process $v(t, x)$.

Then, our main result can be formulated as follows.

Theorem 2.1. Let $u(t, x)$ be the solution (1.2) of the Burgers' equation (1.1) with the initial datum $\xi(x)=\xi_{L}(x), x \in \mathbf{R}$, of the form (2.1) and satisfying conditions (2.3) and (2.4). Then, as $L \rightarrow \infty$, the finite dimensional distributions of $u\left(L^{2} t, L^{2} x\right),(t, x) \in \mathbf{R}_{+} \times \mathbf{R}$, tend to the corresponding distributions of the random field

$$
v(t, x)=\frac{x-y_{J^{*}(t, x)}}{t} .
$$

Here, $y_{j^{*}(t, x)} \equiv y_{j^{*}}$ is the abscissa of the point of a Poisson process $\left(y_{j}, u_{j}\right)_{j \in \mathbf{Z}}$ on $\mathbf{R}^{2}$, with intensity $e^{-u} d u d y$, which maximizes $u_{j}-\left(x-y_{j}\right)^{2} / 2 t$, i.e.

$$
u_{j^{*}}-\frac{\left(x-y_{j^{*}}\right)^{2}}{2 t}=\max _{j}\left(u_{j}-\frac{\left(x-y_{j}\right)^{2}}{2 t}\right) .
$$

The intuitive meaning of Theorem 2.1 can be best explained with the help of the geometric construction presented on Fig. 1 which, actually, goes back to the original Burgers' (1974) work. Also, notice that the limit random field $v(t, x)$ does not depend on the viscosity parameter $\mu$ in Eq. (1.1), and that its shape is what one usually sees in the study of the Burgers' equation in the zero viscosity limit. 
Proof of Theorem 2.1. To simplify the notation, we shall consider only the convergence of one-dimensional distributions of $u\left(L^{2} t, L^{2} x\right)$ for $\mu=t=1 / 2, x=0$. Afterwards, we shall explain how the general case can be obtained.

Put

$$
\begin{aligned}
H_{L} & :=u\left(L^{2} / 2,0\right), \\
a_{L} & :=\sigma_{L} / L^{2}=\sqrt{2 \log L}, \\
b_{L} & :=\sqrt{2 \log L}+\frac{c_{1}}{\sqrt{2 \log L}},
\end{aligned}
$$

where $c_{1}=\log \left(\sqrt{\lambda_{2}} / 2 \pi\right)$. According to (1.2), (2.1),

$$
H_{L}=\frac{-2 \int_{\mathbf{R}} y \exp \left[L^{2}\left(\eta_{L}(y)-y^{2}\right)\right] d y}{\int_{\mathbf{R}} \exp \left[L^{2}\left(\eta_{L}(y)-y^{2}\right)\right] d y},
$$

where

$$
\eta_{L}(y)=a_{L}\left(\eta\left(L_{y}\right)-b_{L}\right) .
$$

Let $y_{j}^{\left(\eta_{L}\right)}, u_{j}^{\left(\eta_{L}\right)}=\eta_{L}\left(y_{j}^{\left(\eta_{L}\right)}\right)$ be positions and heights of local maxima of the process $\eta_{L}(x), x \in \mathbf{R}$, respectively. Due to condition (2.4) of the theorem, their number is a.s. finite on any finite interval (see Leadbetter et al. (1983), Sect. 7.6). Let $\left(y_{j^{*}}^{\left(\eta_{L}\right)}, u_{j^{*}}^{\left(\eta_{L}\right)}\right)$ be the pair which maximizes $u_{j}^{\left(\eta_{L}\right)}-\left(y_{j}^{\left(\eta_{L}\right)}\right)^{2}=\eta_{L}\left(y_{j}^{\left(\eta_{L}\right)}\right)-\left(y_{j}^{\left(\eta_{L}\right)}\right)^{2}, j \in$ $\mathbf{Z}$, i.e.,

$$
u_{j^{*}}^{\left(\eta_{L}\right)}-\left(y_{j^{*}}^{\left(\eta_{L}\right)}\right)^{2}=\max _{j}\left(u_{j}^{\left(\eta_{L}\right)}-\left(y_{J}^{\left(\eta_{L}\right)}\right)^{2}\right) .
$$

(In the case when the last maximum is achieved at several points, we chose the one with the smallest ordinate.) Now, put

$$
I\left(\Delta_{j^{*}}^{\left(\eta_{L}\right)}\right)=\int_{\Delta_{J^{*}}^{\left(\eta_{L}\right)}} \exp \left[L^{2}\left(\eta_{L}(y)-y^{2}\right)\right] d y,
$$

where

$$
\Delta_{j^{*}}^{\left(\eta_{L}\right)}=\left\{y \in \mathbf{R}:\left|y-y_{J^{*}}^{\left(\eta_{L}\right)}\right|<1 / L a_{L}\right\} .
$$

Then, $H_{L}$ of (2.9) can be written as

$$
H_{L}=-2 \frac{y_{j^{*}}^{\left(\eta_{L}\right)}+R_{L}+\rho_{L}}{1+Q_{L}}
$$

where

$$
\begin{aligned}
& R_{L}=\int_{\mathbf{R} \backslash \Delta_{J^{*}}^{\left(\eta_{L}\right)}} y \exp \left[L^{2}\left(\eta_{L}(y)-y^{2}\right)\right] d y / I\left(\Delta_{j^{*}}^{\left(\eta_{L}\right)}\right), \\
& Q_{L}=\int_{\mathbf{R} \backslash \Delta_{J^{*}}^{\left(\eta_{L}\right)}} \exp \left[L^{2}\left(\eta_{L}(y)-y^{2}\right)\right] d y / I\left(\Delta_{j^{*}}^{\left(\eta_{L}\right)}\right),
\end{aligned}
$$

and

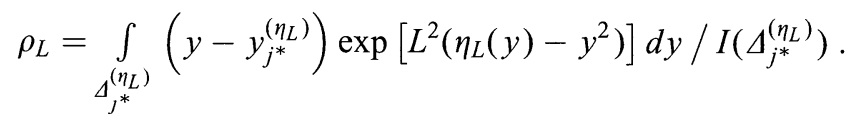


Clearly, the convergence in distribution

$$
H_{L} \Rightarrow v(1 / 2,0)=-2 y_{j^{*}}
$$

follows from the facts that

$$
\begin{aligned}
y_{j^{*}}^{\left(\eta_{L}\right)} & \Rightarrow y_{j^{*}} \equiv y_{j^{*}(1 / 2,0)}, \\
R_{L} & \Rightarrow 0, \\
Q_{L} & \Rightarrow 0,
\end{aligned}
$$

and from the trivial bound $\left|\rho_{L}\right|<2 / L a_{L} \rightarrow 0(L \rightarrow \infty)$.

Proof of the theorem requires a study of the Poisson convergence of functionals of a Gaussian trajectory near high local maxima, in the spirit of Chapter 10 of Leadbetter, Lindgren, Rootzen (1983). Moreover, to prove (2.18) we need a criterion for convergence of the point process $\left(y_{j}^{\left(\eta_{L}\right)}, u_{j}^{\left(\eta_{L}\right)}\right)_{j \in \mathbf{Z}}$ in a topology matched to the Burgers' equation. That topology will be introduced and studied in Sect. 4. The proof of Theorem 2.1 will then be completed in Sect. 5 .

\section{Poisson Convergence of Local Maxima}

Let $\mathscr{M}$ be the space of all locally finite point measures on $\mathbf{R}^{2}$, with the topology of vague convergence of measures, denoted by $\rightarrow$ (see Kallenberg (1983)). Introduce also the space $\overline{\mathscr{M}}$ of all locally finite point measures on $\mathbf{R}^{2}$, taking values in the Banach space $C[-1,1]$ of continuous functions, equipped with the supremum norm. $\|\cdot\|$. Elements $\bar{v} \in \bar{M}$ can be identified with countable sequences

$$
\bar{v}=\left(y_{j}, u_{j}, g_{j}\right)_{j \in \mathbf{Z}},
$$

where $\left(y_{j}, u_{j}\right) \in \mathbf{R}^{2}$ and $g_{j} \in C[-1,1], j \in \mathbf{Z}$. Write $v=\left(y_{j}, u_{j}\right)_{j \in \mathbf{Z}}$. Then $v$ can be identified with the element $\left.\sum_{J \in \mathbf{z}} \delta_{(} y_{j}, u_{j}\right) \in \mathscr{M}$. The convergence $\bar{v}_{L} \rightarrow \bar{v}$ $\left(\bar{v}_{L}, \bar{v} \in \overline{\mathscr{M}}\right)$ is equivalent to the condition that $v_{L} \rightarrow v$ (in $\left.\mathscr{M}\right)$ and that

$$
\left\|g_{j, L}-g_{j}\right\| \rightarrow 0
$$

for any $j \in \mathbf{Z}$. It is clear that $\overline{\mathscr{M}}$, as well as $\mathscr{M}$, are complete, metrizable spaces with respect to the above topology. Without any risk of misunderstanding, we will use the same notation $\Rightarrow$ for the weak convergence of random elements from $\mathscr{M}, \overline{\mathscr{M}}$, and/or from a finite dimensional Euclidean space.

With the Gaussian process $\eta_{L}(x)$ of (2.10) we associate the point process $v^{\left(\eta_{L}\right)}=$ $\left(y_{j}^{\left(\eta_{L}\right)}, u_{j}^{\left(\eta_{L}\right)}\right)_{j \in \mathbf{Z}} \in \mathscr{M}$ of local maxima, and the point process

$$
\bar{v}^{\left(\eta_{L}\right)}=\left(y_{j}^{\left(\eta_{L}\right)}, u_{j}^{\left(\eta_{L}\right)}, g_{J}^{\left(\eta_{L}\right)}\right)_{j \in \mathbf{Z}} \in \overline{\mathscr{M}},
$$

which includes the "germs" $g_{j}^{\left(\eta_{L}\right)}(\cdot) \in C[-1,1]$ of the trajectory near local maxima, where, for $y \in[-1,1]$,

$$
\begin{aligned}
g_{j}^{\left(\eta_{L}\right)}(y) & =\eta_{L}\left(y_{j}^{\left(\eta_{L}\right)}+y / L a_{L}\right)-\eta_{L}\left(y_{j}^{\left(\eta_{L}\right)}\right) \\
& =\eta_{L}\left(y_{j}^{\left(\eta_{L}\right)}+y / L a_{L}\right)-u_{j}^{\left(\eta_{L}\right)} .
\end{aligned}
$$


Proposition 3.1. The point process

$$
\bar{v}^{\left(\eta_{L}\right)} \Longrightarrow \bar{v}
$$

where $\bar{v}=\left(y_{j}, u_{j}, g_{j}\right)_{j \in \mathbf{Z}}$, with $v=\left(y_{j}, u_{j}\right)_{j \in \mathbf{Z}}$ being the Poisson process of Theorem 2.1 , and

$$
g_{j}(y) \equiv g(y)=-\frac{1}{2} \lambda_{2} y^{2}, \quad y \in[-1,1]
$$

being a deterministic parabola.

Proof. The lemma is equivalent to the statement that both

$$
v^{\left(\eta_{L}\right)} \Rightarrow v
$$

and

$$
P-\lim _{L \rightarrow \infty}\left\|g_{J}^{\left(\eta_{L}\right)}-g\right\|=0
$$

for any $j \in \mathbf{Z}$ such that the corresponding local maximum $\left(y_{j}^{\left(\eta_{L}\right)}, u_{J}^{\left(\eta_{L}\right)}\right)$ lies in a fixed compact set $\left[x_{1}, x_{2}\right] \times\left[u_{1}, u_{2}\right] \subset \mathbf{R}^{2}$ for all, sufficiently large $L$. Relation (3.7) is well-known, see e.g. Leadbetter, Lindgren, Rootzen (1983), Theorem 9.5.2. Statement (3.8) can be proved using the Slepian model process representation near a local maximum (due to Lindgren (1970)) as follows. In view of the above, it suffices to prove that, for any $\varepsilon>0$,

$$
\sum_{J} P\left[\left\|g_{J}^{\left(\eta_{L}\right)}-g\right\|>\varepsilon,\left(y_{j}^{\left(\eta_{L}\right)}, u_{j}^{\left(\eta_{L}\right)}\right) \in\left[x_{1}, x_{2}\right] \times\left[u_{1}, u_{2}\right]\right] \rightarrow 0
$$

as $L \rightarrow \infty$. Write the left-hand side as

$$
\sum_{J} E\left[P\left[\left\|g_{j}^{\left(\eta_{L}\right)}-g\right\|>\varepsilon \mid y_{j}^{\left(\eta_{L}\right)}, u_{j}^{\left(\eta_{L}\right)}\right] \cdot \mathbf{1}\left(\left(y_{j}^{\left(\eta_{L}\right)}, u_{j}^{\left(\eta_{L}\right)}\right) \in\left[x_{1}, x_{2}\right] \times\left[u_{1}, u_{2}\right]\right)\right] .
$$

According to Theorem 3 of Lindgren (1970),

$$
\begin{aligned}
& P\left[\left\|g_{J}^{\left(\eta_{L}\right)}-g\right\|>\varepsilon \mid u_{j}^{\left(\eta_{L}\right)}=u, y_{j}^{\left(\eta_{L}\right)}=y\right] \\
& \quad=P\left[\sup _{|x| \leqq 1}\left|a_{L}\left(\eta^{v_{L}}\left(x / a_{L}\right)-v_{L}\right)+\frac{1}{2} \lambda_{2} x^{2}\right|>\varepsilon\right],
\end{aligned}
$$

where $v_{L}=a_{L}+\left(u+c_{1}\right) / a_{L}$ and, for any $v \in \mathbf{R}$,

$$
\eta^{v}(x)=v A(x)+\zeta_{1}(x)-\zeta_{2, v}(x), \quad x \in[-1,1],
$$

is the Slepian model process conditioned at a local maximum of height $v$ at $x=0$. Here $A(x)=\left(\lambda_{4} r(x)+\lambda_{2} r^{\prime \prime}(x)\right) / D, D=\lambda_{4}-\lambda_{2}^{2}>0$, while $\zeta_{1}(x)$ and $\zeta_{2, v}(x)$ are independent stochastic processes with

$$
\zeta_{2, v}(x)=\kappa_{v} B(x)
$$

where $B(x)=\left(\lambda_{2} r(x)+r^{\prime \prime}(x)\right) / D$, and $\kappa_{v}>0$ is a random variable with the density proportional to $z \exp \left[-\left(z-\lambda_{2} v\right)^{2} / 2 D\right], z>0$. The process $\zeta_{1}(x)$ is a zero mean Gaussian process with the covariance function $C(x, y)$ given in Lindgren (1970), 
Eq. (8). Making use of condition (2.4), and the fact that $A(0)=1, A^{\prime}(0)=A^{\prime \prime}(0)=$ 0 , one easily obtains that

$$
a_{L} v_{L}\left(A\left(x / a_{L}\right)-1\right) \rightarrow 0,
$$

uniformly in $x \in[-1,1], u \in\left[u_{1}, u_{2}\right]$. Next, using the fact that $\zeta_{1}(x)$ is a.s. continuously differentiable, and that $\zeta_{1}(0)=\zeta_{1}^{\prime}(0)=0$, similarly as in Leadbetter, Lindgren, Rootzen (1983), p. 203, we conclude that

$$
\sup _{|x| \leqq 1} a_{L}\left|\zeta_{1}\left(x / a_{L}\right)\right| \rightarrow 0, \quad \text { a.s . }
$$

Finally, noting that

$$
\sup _{|x| \leqq 1}\left|-a_{L} v_{L} \lambda_{2} B\left(x / a_{L}\right)+\frac{1}{2} \lambda_{2} x^{2}\right| \rightarrow 0,
$$

and denoting by $p_{L, u}$ the probability in (3.11), we obtain that

$$
p_{L, u}=P\left[\left|1-\left(\kappa_{v_{L}} / \lambda_{2} v_{L}\right)\right|>\varepsilon\right]+o(1) \rightarrow 0,
$$

uniformly in $u \in\left[u_{1}, u_{2}\right]$, as $\kappa_{v} / \lambda_{2} v \rightarrow 1(v \rightarrow \infty)$, in probability. Since $\left(y_{j}^{\left(\eta_{L}\right)}, u_{j}^{\left(\eta_{L}\right)}\right)_{j \in \mathbf{Z}}=v^{\left(\eta_{L}\right)}$ converges to a Poisson limit (see (3.7)), relations (3.10)(3.12) imply (3.8) and the proposition itself. QED

Proposition 3.1 immediately yields the following lower bound for the exponential integral in (2.12).

Corollary 3.1. For any compact $A \subset \mathbf{R}^{2}$, and any $\varepsilon, \delta>0$, there exists an $L_{0}<\infty$ such that, for every $L>L_{0}$,

$$
P\left[I\left(\Delta_{j^{*}}^{\left(\eta_{L}\right)}\right)<\frac{\exp \left[L^{2}\left(u_{j^{*}}^{\left(\eta_{L}\right)}-\left(y_{j^{*}}^{\left(\eta_{L}\right)}\right)^{2}-\delta\right)\right]}{\sqrt{e} L^{2} a_{L}}, \quad\left(y_{j^{*}}^{\left(\eta_{L}\right)}, u_{j^{*}}^{\left(\eta_{L}\right)}\right) \in A\right]<\varepsilon .
$$

\section{Burgers' Topology on Point Measures}

Fix $\alpha_{0}, \beta_{0} \geqq 0$, and consider the subspace $\mathscr{B}=\mathscr{B}_{\alpha_{0}, \beta_{0}} \subset \mathscr{M}$ consisting of all measures $v \in \mathscr{M}$ such that for any $\alpha>\alpha_{0}, \beta>\beta_{0}$,

$$
I_{\alpha, \beta}(v):=\int_{\mathbf{R}^{2}} e^{\alpha u-\beta y^{2}} d v<\infty .
$$

Definition 4.1. Let $v_{L}, v \in \mathscr{B}$. We shall say that $v_{L} \stackrel{\mathscr{B}}{\rightarrow} v$ as $L \rightarrow \infty$, if $v_{L} \rightarrow v$ and

$$
I_{\alpha, \beta}\left(v_{L}\right) \rightarrow I_{\alpha, \beta}(v), \quad L \rightarrow \infty,
$$

for any $\alpha>\alpha_{0}, \beta>\beta_{0}$. The convergence $\stackrel{\mathscr{B}}{\rightarrow}$ defines a topology in $\mathscr{B}$ which henceforth will be called the Burgers' topology ( $\mathscr{B}$-topology).

The next lemma provides a criterion of compactness in the $\mathscr{B}$-topology.

Proposition 4.1. $\mathscr{B}$ is a complete separable metrizable space in the $\mathscr{B}$-topology. $A$ Borel set $A \subset B$ is compact in the $\mathscr{B}$-topology if, and only if, $A$ is compact in the vague topology, and for any $\alpha>\alpha_{0}, \beta>\beta_{0}$,

$$
\sup _{v \in A} I_{\alpha, \beta}(v)<\infty \text {. }
$$


Proof. The lemma follows easily from the well-known properties of $\mathscr{M}$ and of the vague topology (Kallenberg $(1983), 15.7)$, and from the following observation. Let $v_{L} \rightarrow v$ and $\sup _{L}\left(I_{\alpha^{\prime}, \beta^{\prime}}\left(v_{L}\right)+I_{\alpha^{\prime \prime}, \beta^{\prime}}\left(v_{L}\right)\right)<\infty$ for some $\alpha_{0}<\alpha^{\prime}<\alpha<$ $\alpha^{\prime \prime}<\infty, \beta_{0}<\beta^{\prime}<\beta$. Then $I_{\alpha, \beta}(v)<\infty$ and (4.2) holds. Indeed, as $I_{\alpha, \beta}\left(v_{L}\right)<$ $I_{\alpha^{\prime}, \beta^{\prime}}\left(v_{L}\right)+I_{\alpha^{\prime \prime}, \beta^{\prime}}\left(v_{L}\right)$, so $\sup _{L} I_{\alpha, \beta}\left(v_{L}\right)=: \bar{I}_{\alpha, \beta}<\infty$. Assume that $I_{\alpha, \beta}(v)=\infty$. Then, one can find a compact $A \subset \mathbf{R}^{2}$ such that

$$
\int_{A} \exp \left[\alpha u-\beta x^{2}\right] d v>2 \bar{I}_{\alpha, \beta}
$$

On the other hand, from $v_{L} \rightarrow v$ it follows that

$$
\int_{A} e^{\alpha u-\beta x^{2}} d v \leqq \lim \sup \int_{A} e^{\alpha u-\beta x^{2}} d v_{L} \leqq \bar{I}_{\alpha, \beta},
$$

which is a contradiction, i.e. $I_{\alpha, \beta}(v)<\infty$. To prove (4.2), note that, by a similar argument as above, $\lim _{L} \int I_{\alpha, \beta}\left(v_{L}\right) \geqq I_{\alpha, \beta}(v)$. Assume that $\lim _{k} I_{\alpha, \beta}\left(v_{L_{k}}\right)>I_{\alpha, \beta}(v)$ for some sequence $\left\{L_{k}\right\}$; for simplicity take $L_{k}=k$. Then, since $v_{k} \rightarrow v$, one can find $\varepsilon>0$ and a sequence $R_{k} \rightarrow \infty$ such that

$$
i_{k}(\alpha, \beta):=\int_{\left\{|u|+|x|>R_{k}\right\}} e^{\alpha u-\beta x^{2}} d v_{k}>\varepsilon .
$$

Write

$$
i_{k}(\alpha, \beta)=i_{k}^{\prime}(\alpha, \beta)+i_{k}^{\prime \prime}(\alpha, \beta)
$$

where

$$
i_{k}^{\prime}(\alpha, \beta)=\int_{\left\{|u|+|x|>R_{k}, u>0\right\}} e^{\alpha u-\beta x^{2}} d v_{k}
$$

Obviously,

$$
i_{k}^{\prime}(\alpha, \beta) \leqq I_{\alpha^{\prime \prime}, \beta^{\prime}}\left(v_{k}\right) e^{-d_{k}},
$$

where

$$
d_{k}:=\inf \left\{\left(\alpha^{\prime \prime}-\alpha\right) u+\left(\beta-\beta^{\prime}\right) x^{2}: u>0,|u|+|x|>R_{k}\right\} \rightarrow \infty,
$$

as $k \rightarrow \infty$. Since

$$
\sup _{k} I_{\alpha^{\prime \prime}, \beta^{\prime}}\left(v_{k}\right)<\infty,
$$

we have that $\lim _{k} i_{k}^{\prime}(\alpha, \beta)=0$. Similarly, $\lim _{k} i_{k}^{\prime \prime}(\alpha, \beta)=0$, which contradicts (4.4). QED

Let $\mathbf{P}(\mathscr{M}), \mathbf{P}(\mathscr{B})$ denote the family of probability measures on $\mathscr{M}, \mathscr{B}$, respectively. Write $\Rightarrow$ and $\stackrel{\mathscr{B}}{\Rightarrow}$ for the weak convergence of probability measures on, or random elements in, $\mathscr{M}$ and $\mathscr{B}$, respectively. The next proposition provides a characterization of the latter convergence.

Proposition 4.2. Let $P_{L}, P \in \mathbf{P}(\mathscr{B})$. Then $P_{L} \stackrel{\not ด}{\Rightarrow} P$ if, and only if,

(i) $P_{L} \Rightarrow P$, and

(ii) $P_{L} \circ I_{\alpha, \beta}^{-1} \Rightarrow P \circ I_{\alpha, \beta}^{-1}, \quad \forall \alpha>\alpha_{0}, \forall \beta>\beta_{0}$. 
Proof. The necessity of (i) and (ii) for $P_{L} \stackrel{\mathscr{B}}{\Rightarrow} P$ is easy. In particular, (ii) follows from the fact that $I_{\alpha, \beta}(v)$ is continuous on $\mathscr{B}$.

To prove the converse part, it suffices to show that $\left\{P_{L}\right\}$ is tight in $\mathbf{P}(\mathscr{B})$, i.e., that for any $\varepsilon>0$ there exists a compact $K \subset \mathscr{B}$ such that

$$
\sup _{L} P_{L}(\mathscr{B} \backslash K)<\varepsilon .
$$

By (i), there exists a compact $K_{\mathscr{M}} \subset \mathscr{M}$ such that $\sup _{L} P_{L}\left(\mathscr{B} \backslash K_{\mathscr{M}}\right)<\varepsilon / 2$, and by (ii), for any $k \geqq 1$ there exists a compact $C_{k} \subset \mathbf{R}$ such that

$$
\sup _{L}\left(P_{L} \circ I_{\alpha_{0}+k, \beta_{0}+k^{-1}}^{-1}+P_{L} \circ I_{\alpha_{0}+k^{-1}, \beta_{0}+k^{-1}}^{-1}\right)\left(\mathbf{R} \backslash C_{k}\right)<\varepsilon / 2^{k+1} .
$$

Put

$$
K=K_{\mathscr{M}} \cap \bigcap_{k=1}^{\infty}\left(I_{\alpha_{0}+k, \beta_{0}+k^{-1}}^{-1}\left(C_{k}\right) \cap I_{\alpha_{0}+k^{-1}, \beta_{0}+k^{-1}}^{-1}\left(C_{k}\right)\right) .
$$

Then, from Proposition 4.1, we obtain that $K \subset \mathscr{B}$ is compact, and

$$
P_{L}(\mathscr{B} \backslash K) \leqq \frac{\varepsilon}{2}+\sum_{k=1}^{\infty} \frac{\varepsilon}{2^{k+1}}=\varepsilon,
$$

which proves (4.5). QED

Now, we can return to the study of the convergence of the point processes from Lemma 3.1 in the $\mathscr{B}$-topology. Fix $\alpha_{0}=1, \beta_{0}=0$, so that

$$
\mathscr{B}=\mathscr{B}_{1,0}=\left\{v \in \mathscr{M}: I_{\alpha, \beta}(v)<\infty, \quad \forall \alpha>1, \beta>0\right\} .
$$

Proposition 4.3. For any $L>0$, the point processes $v^{\left(\eta_{L}\right)}$ and $v$ of Lemma 3.1 belong to $\mathscr{B}$ a.s. and, as $L \rightarrow \infty$,



Proof. The relation $v=\left(y_{J}, u_{j}\right)_{j \in \mathbf{Z}} \in \mathscr{B}$ a.s. follows from the facts that

$$
\iint_{\left\{\alpha u-\beta y^{2}>0\right\}} e^{-u} d u d y=\int_{-\infty}^{\infty} e^{-(\beta / \alpha) y^{2}} d y<\infty,
$$

and

$$
\iint_{\left\{\alpha u-\beta y^{2}<0\right\}} e^{\alpha u-\beta y^{2}} e^{-u} d u d y=\frac{1}{\alpha-1} \int_{-\infty}^{\infty} e^{-(\beta / \alpha) y^{2}} d y<\infty
$$

(see e.g. Kwapien, Woyczynski (1992), Chapters 7 and 8). According to Proposition 3.1 and Proposition 4.2, the $\mathscr{B}$-convergence in (4.6) will follow once we demonstrate that, as $L \rightarrow \infty$, the (one-dimensional) distributions of

$$
I_{\alpha, \beta}\left(v^{\left(\eta_{L}\right)}\right) \Longrightarrow I_{\alpha, \beta}(v)
$$

for any $\alpha>1, \beta>0$, where

$$
I_{\alpha, \beta}\left(v^{\left(\eta_{L}\right)}\right)=\sum_{j \in \mathbf{Z}} \exp \left[\alpha u_{j}^{\left(\eta_{L}\right)}-\beta\left(y_{j}^{\left(\eta_{L}\right)}\right)^{2}\right]=\int_{\mathbf{R}^{2}} e^{\alpha u-\beta y^{2}} d v^{\left(\eta_{L}\right)} .
$$


We shall prove (4.7) by first decomposing

$$
I_{\alpha, \beta}\left(v^{\left(\eta_{L}\right)}\right)=\sum_{p=1}^{3} J_{p}\left(v^{\left(\eta_{L}\right)}\right)
$$

where

$$
\begin{aligned}
& J_{1}\left(v^{\left(\eta_{L}\right)}\right)=\int_{A_{\alpha, \beta, \gamma} \cap\{u<K\}} e^{\alpha u-\beta y^{2}} d v^{\left(\eta_{L}\right)}, \\
& J_{2}\left(v^{\left(\eta_{L}\right)}\right)=\int_{A_{\alpha, \beta, \gamma} \cap\{u>K\}} e^{\alpha u-\beta y^{2}} d v^{\left(\eta_{L}\right)}, \\
& J_{3}\left(v^{\left(\eta_{L}\right)}\right)=\int_{A_{\alpha, \beta, i}^{c}} e^{\alpha u-\beta y^{2}} d v^{\left(\eta_{L}\right)},
\end{aligned}
$$

and where

$$
A_{\alpha, \beta, \gamma}=\left\{(y, u) \in \mathbf{R}^{2}: \alpha u-\beta y^{2}+\gamma>0\right\}
$$

is the set above the parabola

$$
\partial A_{\alpha, \beta, \gamma}=\left\{(y, u): \alpha u-\beta y^{2}+\gamma=0\right\},
$$

$\gamma \in \mathbf{R}^{2} ; A_{\alpha, \beta, \gamma}^{c}=\mathbf{R}^{2} \backslash A_{\alpha, \beta, \gamma}$.

From Lemma 3.1 one easily obtains that, for any $\gamma, K \in \mathbf{R}$,

$$
J_{1}\left(v^{\left(\eta_{L}\right)}\right) \Rightarrow J_{1}(v)=\int_{A_{\alpha, \beta, \gamma} \cap\{u<K\}} e^{\alpha u-\beta y^{2}} d v,
$$

as $L \rightarrow \infty$. Moreover, as $\gamma, K \rightarrow \infty$,

$$
J_{1}(v) \Rightarrow I_{\alpha, \beta}(v) \text {. }
$$

Therefore (4.7) will follow once we prove that

$$
P-\lim _{\gamma \rightarrow \infty} J_{3}\left(v^{\left(\eta_{L}\right)}\right)=0
$$

and that, for any $\gamma \in \mathbf{R}$,

$$
P-\lim _{K \rightarrow \infty} J_{2}\left(v^{\left(\eta_{L}\right)}\right)=0
$$

uniformly in $L \rightarrow \infty$.

Let us begin with the proof of convergence of $J_{2}$ in (4.10). Write

$$
\begin{aligned}
J_{2}\left(v^{\left(\eta_{L}\right)}\right) & =\sum_{k=-\infty}^{\infty} \int_{A_{\alpha \beta, \gamma} \cap\{u>K, y \in(j, j+1]\}} e^{\alpha u-\beta y^{2}} d v^{\left(\eta_{L}\right)} \\
& \equiv \sum_{k=-\infty}^{\infty} \xi_{j, K, L} .
\end{aligned}
$$

Here, for $j>0$ and $L>1$,

$$
\begin{aligned}
P\left[\xi_{j, K, L}>0\right] & \leqq P\left[\max _{x \in(j, j+1]} \eta_{L}(x)>\left(\left(\beta j^{2}-\gamma\right) / \alpha\right) \vee K\right] \\
& \leqq C_{1} \exp \left[-\left(\left(\beta j^{2}-\gamma\right) / \alpha \vee K\right)\right],
\end{aligned}
$$


with $C_{1}<\infty$ independent of $j, K, L$, and other parameters (see Leadbetter, Lindgren Rootzen (1983)). A similar estimate clearly holds for $j \leqq 0$. Consequently, for every $L>1$,

$$
P\left[J_{2}\left(v^{\left(\eta_{L}\right)}\right)>0\right] \leqq \sum_{j=-\infty}^{\infty} P\left[\xi_{j, K, L}>0\right]=O\left(e^{-c_{2} K}\right)
$$

with $c_{2}=c_{2}(\alpha, \beta, \gamma)>0$, which proves $(4.10)$.

Next, we shall prove (4.9). Here, the expectations exist, so we can work directly with them rather than with probabilities which were needed in the analysis of $J_{2}$.

According to Leadbetter, Lindgren, Rootzen (1983), p. 161,

$$
E v^{\left(\eta_{L}\right)}(d y, d u)=\Gamma_{L}(u) e^{-u} d u d y,
$$

where, with $c_{1}=\log \left(\sqrt{\lambda_{2}} / 2 \pi\right)$ and $D=\lambda_{4}-\lambda_{2}^{2}>0$,

$$
\begin{aligned}
\Gamma_{L}(u)= & \exp \left[-\lambda_{4}\left(u+c_{1}\right)^{2} / 2 D a_{L}^{2}\right] \\
& \times\left(1+\left(1 / \lambda_{2} a_{L} \sqrt{2 \pi D}\right) \int_{-\infty}^{\infty}\left(x \vee\left(-a_{L} \lambda_{2}\right)\right)\right. \\
& \left.\exp \left[-x^{2} / 2 D+\lambda_{2}\left(u+c_{1}\right) x / D a_{L}\right] d x\right)
\end{aligned}
$$

From there, it easily follows that

$$
\begin{aligned}
\Gamma_{L}(u) \leqq & \exp \left[-\left(u+c_{1}\right)^{2} / 2 a_{L}^{2}\right] \\
& \times\left(1+\left(1 / \lambda_{2} a_{L} \sqrt{2 \pi D}\right) \int_{-\infty}^{\infty}\left(\left(z+\frac{\lambda_{2}\left(u+c_{1}\right)}{a_{L}}\right) \vee\left(-a_{L} \lambda_{2}\right)\right) e^{-z^{2} / 2 D} d z\right) \\
< & C,
\end{aligned}
$$

with $C<\infty$ and independent of $L$, and $u \in \mathbf{R}$. Therefore

$$
\begin{aligned}
E J_{3}\left(v^{\left(\eta_{L}\right)}\right) & =\int_{A_{\alpha, \beta, \gamma}^{c}} e^{\alpha u-\beta y^{2}} \Gamma_{L}(u) e^{-u} d u d y \\
& \leqq C \int_{A_{\alpha, \beta, \gamma}^{c}} e^{\alpha u-\beta y^{2}} e^{-u} d u d y \\
& =\frac{C}{\alpha-1} e^{-\gamma(\alpha-1) / \alpha} \int_{-\infty}^{\infty} e^{-(\beta / \alpha) y^{2}} d y \rightarrow 0,
\end{aligned}
$$

and $\gamma \rightarrow \infty$, uniformly in $L>1$. This proves (4.9) and the Proposition 4.3 as well. QED

Below we use Proposition 4.3 to prove the convergence (2.18) of the local maximum point $\left(y_{j^{*}}^{\left(\eta_{L}\right)}, u_{j^{*}}^{\left(\eta_{L}\right)}\right)$.

Proposition 4.4. As $L \rightarrow \infty$,

$$
\left(y_{J^{*}}^{\left(\eta_{L}\right)}, u_{j^{*}}^{\left(\eta_{L}\right)}\right) \Rightarrow\left(y_{j^{*}}, u_{j^{*}}\right)
$$

where $\left(y_{j^{*}}, u_{j^{*}}\right)$ is the Poisson point process described in Theorem 2.1. In particular,

$$
u_{j^{*}}^{\left(\eta_{L}\right)}-\left(y_{j^{*}}^{\left(\eta_{L}\right)}\right)^{2} \Rightarrow u_{j^{*}}-y_{j^{*}}^{2}
$$


Proof. Consider the set $\mathscr{B}_{0}=\mathscr{B} \cap \mathscr{M}_{0}$, where $\mathscr{M}_{0}$ is the set of all simple point measures $v \in \mathscr{M}$. Each $v \in \mathscr{B}_{0}$ can be identified with its range, that is, a locally finite, countable set $\cup_{j \in \mathbf{Z}}\left\{\left(y_{j}, u_{j}\right)\right\}$ (without any danger of misunderstanding we shall use for the latter the same notation $\left.v=\left(y_{j}, u_{j}\right)_{j \in \mathbf{Z}}\right)$. Note, that for any "parabolic" set $A_{\alpha, \beta, \gamma}, \alpha>1, \beta>0, \gamma \in \mathbf{R}$ (see (4.8)), and any $v \in \mathscr{B}_{0}$ such that $v\left(\partial A_{\alpha, \beta, \gamma}\right)=0$, we have that $v_{L} \stackrel{\not B}{\rightarrow} v$ implies $v_{L}\left(A_{\alpha, \beta, \gamma}\right)=v\left(A_{\alpha, \beta, \gamma}\right)$ for all sufficiently large $L$, and that

$$
\operatorname{dist}\left(v_{L} \cap A_{\alpha, \beta, \gamma}, v \cap A_{\alpha, \beta, \gamma}\right) \rightarrow 0 \text {. }
$$

Consider the functional

$$
h(v)=\left(y_{j^{*}}, u_{j^{*}}\right), \quad v=\left(y_{j}, u_{j}\right)_{j \in \mathbf{Z}},
$$

with values in $\mathbf{R}^{2}$, where $j^{*}=j^{*}(1 / 2,0)$. Then $h(v)$, which is clearly not continuous in the usual vague topology, is a.e. well defined and continuous on $\mathscr{B}_{0}$ in the $\mathscr{B}$-topology, with respect to the Poisson measure of Theorem 2.1. Indeed, for every $\alpha>1$ and almost all $v \in \mathscr{B}_{0}$ one can find $\gamma \in \mathbf{R}$ such that $h(v) \in A_{\alpha, \alpha, \gamma}$ and $v\left(\partial A_{x, \alpha, \gamma}\right)=0$. Moreover, for a.e. $v=\left(y_{j}, u_{j}\right)_{j \in \mathbf{Z}} \in \mathscr{B}_{0}$, the maximum on the right-hand side of (2.6) is attained at a single point $\left(y_{j^{*}}, u_{j^{*}}\right) \in v$. Hence, if $v_{L} \stackrel{\mathscr{B}}{\rightarrow} v, v_{L}=\left(y_{j, L}, u_{j, L}\right)_{j \in \mathbf{Z}}$, then (4.13) clearly implies that $h\left(v_{L}\right) \rightarrow h(v)$, i.e., the functional $h(v)$ is a.e. $\mathscr{B}$-continuous. Now, (4.11) and (4.12) follow from Proposition 4.1 and from the well-known properties of the weak convergence.

\section{Proof of Theorem 2.1 (continued)}

Let us return to the proof of convergence in (2.18)-(2.20) of Sect. 2. Consider the set $\Omega_{\delta, \gamma, K, L}$ of points $\omega \in \Omega$ satisfying the following four conditions:

$$
\begin{gathered}
\left(y_{J^{*}}^{\left(\eta_{L}\right)}, u_{J^{*}}^{\left(\eta_{L}\right)}\right) \in A_{2,2, \gamma}, \\
\left(y, \eta_{L}(y)\right) \notin A_{2,1, \gamma}, \quad \text { for }|y|>K, \\
I\left(\Delta_{j^{*}}^{\left(\eta_{L}\right)}\right)>\left(1 / \sqrt{e} L^{2} a_{L}\right) \exp \left[L^{2}\left(u_{j^{*}}^{\left(\eta_{L}\right)}-\left(y_{j^{*}}^{\left(\eta_{L}\right)}\right)^{2}-\delta\right)\right], \\
\eta_{L}(y)-y^{2}<u_{J^{*}}^{\left(\eta_{L}\right)}-\left(y_{j^{*}}^{\left(\eta_{L}\right)}\right)^{2}-2 \delta, \quad \text { for every } y \notin \Delta_{j^{*}}^{\left(\eta_{L}\right)},|y| \leqq K .
\end{gathered}
$$

Then, in view Proposition 4.4, Proposition 4.3, Corollary 3.1 and Proposition 3.1 (in that order), for any $\varepsilon>0, \delta>0$ we can find $\gamma>2 \delta, K<\infty$, and $L_{0}>0$ such that for all $L>L_{0}$,

$$
P\left[\Omega_{\delta, \gamma, K, L}\right]>1-\varepsilon .
$$

Therefore, it suffices to prove (2.18)-(2.20) with $R_{L}$, and $Q_{L}$ replaced by $R_{L}^{\prime}=$ $R_{L} \mathbf{1}\left(\omega \in \Omega_{\delta, \gamma, K, L}\right)$ and $Q_{L}^{\prime}=Q_{L} \mathbf{1}\left(\omega \in \Omega_{\delta, \gamma, K, L}\right)$, respectively. In view of (5.2), $(5.3)$

$$
\begin{aligned}
Q_{L}^{\prime} \leqq & \sqrt{e} L^{2} a_{L}\left(K e^{-L^{2} \delta}\right. \\
& \left.+\exp \left[L^{2}\left(u_{j^{*}}^{\left(\eta_{L}\right)}-\left(y_{j^{*}}^{\left(\eta_{L}\right)}\right)^{2}-\delta\right)\right] \int_{\left\{|y|>K,\left(y, \eta_{L}(y)\right) \notin A_{2,1, \gamma}\right\}} \exp \left[L^{2}\left(\eta_{L}(y)-y^{2}\right)\right] d y\right) \\
\leqq & \sqrt{e} L^{2} a_{L} e^{-L^{2} \delta}\left(K+\int_{\mathbf{R}} e^{-L^{2} y^{2} / 2} d y\right)=O\left(e^{-L^{2} \delta / 2}\right),
\end{aligned}
$$


which proves (2.20). In a similar way, one can prove (2.19). Finally, (2.18) follows from Proposition 4.4. This proves (2.17).

We complete the proof of Theorem 2.1 with a few remarks about the convergence of general finite-dimensional distributions, i.e.

$$
\left(u\left(L^{2} t_{1}, L^{2} x_{1}\right), \ldots, u\left(L^{2} t_{n}, L^{2} x_{n}\right)\right) \Longrightarrow\left(v\left(t_{1}, x_{1}\right), \ldots, v\left(t_{n}, x_{n}\right)\right)
$$

for any $n \geqq 1$, and any points $\left(t_{l}, x_{i}\right) \in \mathbf{R}_{+} \times \mathbf{R}, i=1, \ldots, n$.

Similarly as in (2.13), we write

$$
u\left(L^{2} t_{i}, L^{2} x_{i}\right)=\left(\left(x_{i}-y_{j_{i}^{*}}^{\left(\eta_{L}\right)}\right) / t_{i}+R_{L, i}+\rho_{L, i}\right) /\left(1+Q_{L, i}\right),
$$

$i=1, \ldots, n$, where

$$
\begin{gathered}
u_{j_{i}^{*}}^{\left(\eta_{L}\right)}-\frac{1}{2 t_{i_{i}}}\left(x_{i}-y_{j_{l}^{*}}^{\left(\eta_{L}\right)}\right)^{2}=\max _{j}\left(u_{j}^{\left(\eta_{L}\right)}-\frac{1}{2 t_{i}}\left(x_{i}-y_{j}^{\left(\eta_{L}\right)}\right)^{2}\right), \\
R_{L, i}=\int_{\mathbf{R} \backslash \Delta_{J_{i}^{*}}^{\left(\eta_{L}\right)}} \frac{x_{i}}{t_{i}} \exp \left[L^{2}\left(\eta_{L}(y)-\left(x_{l}-y\right)^{2} / 2 t_{i}\right)\right] d y / I\left(\Delta_{j_{l}^{*}}^{\left(\eta_{L}\right)}\right), \\
Q_{L, i}=\int_{\mathbf{R} \backslash \Delta_{j_{i}^{*}}^{\left(\eta_{L}\right)}} \exp \left[L^{2}\left(\eta_{L}(y)-\left(x_{i}-y\right)^{2} / 2 t_{i}\right)\right] d y / I\left(\Delta_{j_{l}^{*}}^{\left(\eta_{L}\right)}\right), \\
\rho_{L, i}=\int_{\Delta_{j_{i}^{*}}^{\left(\eta_{L}\right)}} \frac{y-y_{j_{l}^{*}}^{\left(\eta_{L}\right)}}{t_{i}} \exp \left[L^{2}\left(\eta_{L}(y)-\left(x_{l}-y\right)^{2} / 2 t_{i}\right)\right] d y / I\left(\Delta_{j_{i}^{*}}^{\left(\eta_{L}\right)}\right),
\end{gathered}
$$

and

$$
\begin{aligned}
\Delta_{j_{l}^{*}}^{\left(\eta_{L}\right)} & =\left\{y \in \mathbf{R}:\left|y-y_{j_{l}^{*}}^{\left(\eta_{L}\right)}\right|<1 / L a_{L}\right\}, \\
I\left(\Delta_{J_{l}^{*}}^{\left(\eta_{L}\right)}\right) & =\int_{\substack{\left(\eta_{j_{L}}^{*}\right) \\
j_{l}^{*}}} \exp \left[L^{2}\left(\eta_{L}(y)-\left(x_{i}-y\right)^{2} / 2 t_{i}\right)\right] d y .
\end{aligned}
$$

Then, similarly as in the proof of (2.17), one can show that for any $i=1, \ldots, n$,

$$
P-\lim _{L \rightarrow \infty}\left|R_{L, i}\right|+Q_{L, i}+\left|\rho_{L, i}\right|=0,
$$

so that (5.5) follows from the fact that

$$
\left(y_{j_{l}^{*}}^{\left(\eta_{L}\right)}, \ldots, y_{j_{i}^{*}}^{\left(\eta_{L}\right)}\right) \Longrightarrow\left(y_{j_{1}^{*}}, \ldots, y_{j_{n}^{*}}\right)
$$

where $j_{i}^{*}=j^{*}\left(t_{l}, x_{l}\right)$.

Consider a functional $\vec{h}(v)=\left(h_{1}(v), \ldots, h_{n}(v)\right), v \in \mathscr{B}$, taking values in $\left(\mathbf{R}^{2}\right)^{n}$, where, for every $i=1, \ldots, n$,

$$
h_{l}(v)=\left(y_{j_{l}^{*}}, u_{j_{l}^{*}}\right) \in v=\left(y_{j}, u_{j}\right)_{j \in \mathbf{Z}} .
$$

In the same way as in Proposition 4.4 one can show that $\vec{h}(v)$ is a.s. well defined and continuous in the $\mathscr{B}$-topology. Hence, in view of Proposition 4.3,

$$
\vec{h}\left(v^{\left(\eta_{L}\right)}\right) \Rightarrow \vec{h}(v)
$$

as $L \rightarrow \infty$, which proves (5.6).

This concludes the proof of Theorem 2.1. QED 


\section{Densities and Correlation Functions of the Limit Velocity Field}

Using the Poisson process representation (2.5) of the limit velocity field $v(t, x)$, one can obtain explicit formulas for its time-space multipoint distribution and correlation functions. Note first that for $n>1$, the joint distribution of

$$
\left(v\left(t_{1}, x_{1}\right), \ldots, v\left(t_{n}, x_{n}\right)\right)
$$

is not absolutely continuous in $\mathbf{R}^{n}$ but rather a sum of absolutely continuous distributions on some $k$-dimensional hyperplanes of $\mathbf{R}^{n}, 1 \leqq k \leqq n$. This is due, of course, to the fact that $P\left[y_{j^{*}\left(t_{i}, x_{i}\right)}=y_{j^{*}\left(t_{l}, x_{l}\right)}\right]>0$ for $i \neq j$.

It follows from (2.5) that both the distribution of (6.1), and its joint moment ( $n$-point correlation function) $\rho^{(n)}\left(t_{1}, x_{1}, \ldots, t_{n}, x_{n}\right)=E v\left(t_{1}, x_{1}\right) \ldots v\left(t_{n}, x_{n}\right)$, can be obtained from the distribution

$$
P^{*}\left(\cdot ;(t, x)_{n}\right)=P\left[\left(y^{*}\right)_{n} \in \cdot\right]
$$

of the random vector

$$
\left(y^{*}\right)_{n}=\left(y_{1}^{*}, \ldots, y_{n}^{*}\right),
$$

where

$$
y_{l}^{*}=y_{j_{l}^{*}} \equiv y_{j^{*}\left(t_{l}, x_{l}\right)},
$$

$i=1, \ldots, n$, and we use the notation

$$
\begin{aligned}
(y)_{n} & =\left(y_{1}, \ldots, y_{n}\right) \in \mathbf{R}^{n}, \\
(t, x)_{n} & =\left(\left(t_{1}, x_{1}\right), \ldots,\left(t_{n}, x_{n}\right)\right) \in\left(\mathbf{R}_{+} \times \mathbf{R}\right)^{n} .
\end{aligned}
$$

In particular,

$$
\rho^{(n)}(t, x)_{n}=\int_{\boldsymbol{R}^{n}} \prod_{i=1}^{n} \frac{x_{t}-y_{t}}{t_{l}} P^{*}\left(d(y)_{n} ;(t, x)_{n}\right) .
$$

We have

$$
P^{*}\left(\cdot ;(t, x)_{n}\right)=\sum_{(A)_{m}} P_{(A)_{m}}^{*}\left(\cdot ;(t, x)_{n}\right)
$$

where the sum is taken over all partitions $(A)_{m}=\left(A_{1}, \ldots, A_{m}\right)$ of $\{1, \ldots, n\}, A_{i} \neq \emptyset$, $A_{i} \cap A_{J}=\emptyset(i \neq j), \bigcup_{l=1}^{m} A_{l}=\{1, \ldots, n\}, m=1, \ldots, n$, and $P_{(A)_{m}}^{*}\left(d(y) m ;(t, x)_{n}\right)$ is a measure on $\mathbf{R}^{m}$ which can be identified with the distribution of $\left(y^{*}\right)_{n}$ on the $m$ dimensional hyperplane

$$
y_{i}^{*}=y_{k}, \quad i \in A_{k}, \quad k=1, \ldots, m .
$$

Note that the last event occurs if, and only if, for every $k=1, \ldots, m$, and any Poisson point $\left(y_{j}, u_{j}\right), j \neq k$, the following inequality is true:

$$
u_{j}<\bigwedge_{i \in A_{k}} g_{i k}\left(y_{j}\right)
$$

where

$$
g_{i k}(y)=u_{k}+\frac{1}{2 t_{l}}\left(\left(y-x_{l}\right)^{2}-\left(y_{k}-x_{i}\right)^{2}\right)
$$

is the parabola passing through the point $\left(y_{k}, u_{k}\right)$ and "centered" at $x_{i}, i \in A_{k}$. Using the well-known formula for the Poisson probabilities, we obtain from (6.7) that for 
each partition $(A)_{m}$, the measure $P_{(A)_{m}}^{*}\left(\cdot ;(t, x)_{n}\right)$ is absolutely continuous in $\mathbf{R}^{m}$, and that the corresponding Radon-Nikodym density is given by

$$
p_{(A)_{m}}^{*}\left((y)_{m} ;(t, x)_{n}\right)=\int_{W(y)_{m}} \exp \left[-\sum_{i=1}^{m} u_{i}-\int \bigvee_{R}^{m} \bigvee_{k=1}^{m} e^{-g_{l k}(z)} d z\right] d(u)_{m},
$$

where

$$
W(y)_{m}=\left\{(u)_{m} \in \mathbf{R}^{m}: u_{j}<\bigwedge_{i \in A_{k}} g_{i k}\left(y_{j}\right), \text { for all } j \neq k, j, k=1, \ldots, m\right\} .
$$

For $n=1,2$ formulas (6.4), (6.9) can be made much more explicit. Consider first a 1-dimensional distribution of $v(t, x)$. Then, according to (6.9) (with $\left.n=m=1,(t, x)_{1}=(t, x),(y)_{1}=y, W(y)_{1}=\mathbf{R}\right)$, we obtain

$$
\begin{aligned}
p^{*}(y ;(t, x)) & =\int_{\mathbf{R}} \exp \left[-u-\int_{\mathbf{R}} \exp \left[-u-\left((z-x)^{2}-(y-x)^{2}\right) / 2 t\right] d z\right] d u \\
& =\int_{\mathbf{R}} \exp \left[-u-\exp \left[-u+(y-x)^{2} / 2 t\right] \sqrt{2 \pi t}\right] d u \\
& =(1 / \sqrt{2 \pi t}) e^{-(y-x)^{2} / 2 t}
\end{aligned}
$$

i.e., $y_{j^{*}(t, x)}$ is Gaussian with parameters $x$, $t$, and consequently, $v(t, x)$ is Gaussian with parameters $0,1 / t$.

Consider the case $n=2,(t, x)_{2}=\left(\left(t_{1}, x_{1}\right),\left(t_{2}, x_{2}\right)\right)$. There are two partitions of $\{1,2\}$, namely, $(A)_{1}=\{1,2\}$, and $(A)_{2}=(\{1\},\{2\})$. From (6.9), we have

$$
p_{1}^{*}(y) \equiv p_{(A)_{1}}^{*}\left(y ;(t, x)_{2}\right)=\left[B_{1}(0 ; y, y)+B_{2}(0 ; y, y)\right]^{-1}
$$

and

$$
\begin{aligned}
p_{2}^{*}\left(y_{1}, y_{2}\right) \equiv & p_{(A)_{2}}^{*}\left(y_{1}, y_{2} ;(t, x)_{2}\right) \\
& =\int_{a_{1}}^{a_{2}}\left[e^{-v / 2} B_{1}\left(v ; y_{1}, y_{2}\right)+e^{v / 2} B_{2}\left(v ; y_{1}, y_{2}\right)\right]^{-2} d v
\end{aligned}
$$

where

$$
\begin{aligned}
a_{l} & =\frac{1}{2 t_{i}}\left(\left(y_{2}-x_{i}\right)^{2}-\left(y_{1}-x_{i}\right)^{2}\right), \\
B_{i}\left(v ; y_{1}, y_{2}\right) & =e^{\left(y_{i}-x_{i}\right)^{2} / 2 t_{i}} \int_{C_{i}\left(v ; y_{1}, y_{2}\right)} e^{-\left(z-x_{i}\right)^{2} / 2 t_{l}} d z, \\
C_{1}\left(v ; y_{1}, y_{2}\right) & =\left\{z \in \mathbf{R}: \frac{\left(z-y_{1}\right)\left(z+y_{1}+2 x_{1}\right)}{2 t_{1}}-\frac{\left(z-y_{2}\right)\left(z+y_{2}+2 x_{2}\right)}{2 t_{2}}<v\right\},
\end{aligned}
$$

and

$$
C_{2}\left(v ; y_{1}, y_{2}\right)=\mathbf{R} \backslash C_{1}\left(v ; y_{1}, y_{2}\right) .
$$

Also, we are able to compute the time-space covariance

$$
\begin{aligned}
\rho^{(2)}\left(t_{1}, x_{1} ; t_{2}, x_{2}\right)= & E v\left(t_{1}, x_{1}\right) v\left(t_{2}, x_{2}\right) \\
= & \frac{1}{t_{1} t_{2}} \int\left(x_{1}-y\right)\left(x_{2}-y\right) p_{1}^{*}(y) d y \\
& +\frac{1}{t_{1} t_{2}} \int\left(x_{\mathbf{R}^{2}}-y_{1}\right)\left(x_{2}-y_{2}\right) p_{2}^{*}\left(y_{1}, y_{2}\right) d y_{1} d y_{2} .
\end{aligned}
$$


Let $t_{1}<t_{2}$; the case $t_{1}=t_{2}$ is simpler and should be treated separately. By the translation invariance of the Poisson process, $\rho^{(2)}\left(t_{1}, x_{1} ; t_{2}, x_{2}\right)=\rho^{(2)}\left(t_{1}, t_{2} ; x_{2}-x_{1}\right)$, and we can assume, without loss of generality, that $x_{1}, x_{2}$ satisfy

$$
x_{1}=\frac{\left(x_{2}-x_{1}\right) t_{1}}{t_{2}-t_{1}}, \quad x_{2}=\frac{\left(x_{2}-x_{1}\right) t_{2}}{t_{2}-t_{1}}
$$

or $x_{1} / t_{1}=x_{2} / t_{2}$. Substituting (6.13) into (6.11) and (6.12), after some elementary, but tedious, transformations we arrive at the formula

$$
\begin{aligned}
\rho^{(2)}\left(t_{1}, x_{1} ; t_{2}, x_{2}\right)= & \frac{1}{t_{1} t_{2}} \int_{-\infty}^{\infty}\left(z-x_{1}\right)\left(z-x_{2}\right) A^{-1}\left(z ; x_{2}, x_{2}\right) d z \\
& -\frac{t-t_{1}}{t_{1} t_{2}} \int_{-\infty}^{\infty}|z|\left(1-e^{-2\left(x_{2}-x_{1}\right) z /\left(t_{2}-t_{1}\right)}\right)^{2} A^{-2}\left(z ; x_{1}, x_{2}\right) d z,
\end{aligned}
$$

where

$$
\begin{aligned}
A\left(z ; x_{1}, x_{2}\right)= & e^{\left(z-x_{1}\right)^{2} / 2 t_{1}} \int_{|y|<|z|} e^{-\left(y-x_{1}\right)^{2} / 2 t_{1}} d y \\
& +e^{\left(z-x_{2}\right)^{2} / 2 t_{2}} \int_{|y|>|z|} e^{-\left(y-x_{2}\right)^{2} / 2 t_{2}} d y .
\end{aligned}
$$

The corresponding expression for fixed time $\left(t_{1}=t_{2} \equiv t\right)$ covariance was obtained in Gurbatov, Malachov, Saichev (1991) p. 181, and is somewhat simpler, namely

$$
\rho^{(2)}\left(t, x_{1} ; t, x_{2}\right)=\frac{1}{t} \frac{d}{d x}\left(x P_{t}(x)\right)
$$

where

$$
P_{t}(2 x)=(1 / \sqrt{2 \pi t}) \int_{-\infty}^{\infty}\left[e^{(x+z)^{2} / 2 t} \Phi_{t}(x+z)+e^{(x-z)^{2} / 2 t} \Phi_{t}(x-z)\right]^{-1} d z
$$

with, as usual, $\Phi_{t}(x)=(1 / \sqrt{2 \pi t}) \int_{-\infty}^{x} e^{-u^{2} / 2 t} d u$, being the probability that the points $x_{1}, x_{2}, x_{2}-x_{1}=2 x$, belong to the same line segment of continuity of the sawtooth process $v(t, x)$ (see Gurbatov, Malachov, Saichev (1991), pp. 175-181, for details).

\section{References}

1. Albeverio, S., Molchanov, S.A., Surgailis, D.: Stratified structure of the Universe and Burgers' equation: A probabilistic approach. Prob. Theory Rel. Fields (1995), to appear

2. Bulinskii, A.V., Molchanov, S.A.: Asymptotic Gaussianness of solutions of the Burgers' equation with random initial data. Teorya Veroyat. Prim. 36, 271-235 (1991)

3. Burgers, J.: The Nonlinear Diffusion Equation. Amsterdam: Dordrecht (1974)

4. Chorin, A.J.: Lectures on Turbulence Theory. Berkeley CA: Publish or Perish (1975)

5. Fournier, J.-D., Frisch, U.: L'équation de Burgers déterministe et statistique. J. Mec. Theor. Appl. 2, 699-750 (1983)

6. Funaki, T., Surgailis, D., Woyczynski, W.A.: Gibbs-Cox random fields and Burgers' turbulence. Ann. Applied Probability 5, to appear (1995)

7. Gurbatov, S., Malakhov, A., Saichev, A.: Nonlinear Random Waves and Turbulence in Nondispersive Media: Waves, Rays and Particles. Manchester: Manchester University Press (1991)

8. Hopf, E.: The partial differential equation $u_{t}+u u_{x}=\mu u_{x x}$. Comm. Pure Appl. Math. 3, 201 (1950) 
9. Hu, Y., Woyczynski, W.A.: An extremal rearrangement property of statistical solutions of the Burgers' equation, Ann. Applied Probability 4, 838-858 (1994)

10. Kallenberg, O.: Random Measures. New York: Academic Press (1983)

11. Kraichnan, R.H.: Lagrangian-history statistical theory for Burgers' equation. Physics of Fluids 11, 265-277 (1968)

12. Kraichnan, R.H.: The structure of isotropic turbulence at very high Reynolds numbers. J. Fluid Mech. 5, 497-543 (1959)

13. Kwapien, S., Woyczynski, W.A.: Random Series and Stochastic Integrals: Single and Multiple, Boston: Birkhäuser (1992)

14. Leadbetter, M.R., Lindgren, G., Rootzen H.: Extremes and Related Properties of Random Sequences and Processes. Berlin, Heidelberg, New York: Springer (1983)

15. Lindgren, G.: Some properties of a normal process mear a local maximum. Ann. Math. Stat. 41, 1870-1883 (1970)

16. Rice S.O.: Mathematical analysis of random noise. Bell System Tech. J. 24, 46-156 (1945)

17. Shandarin, S.F., Zeldovich, Ya.B.: Turbulence, intermittency, structures in a self-gravitating medium: The large scale structure of the Universe. Rev. Modern Phys. 61, 185-220 (1989)

18. Sinai, Ya.G.: Two results concerning asymptotic behavior of solutions of the Burgers equation with force. J. Stat. Phys. 64, 1-12 (1992)

19. Sinai, Ya.G.: Statistics of shocks in solutions of inviscid Burgers' equation. Commun. Math. Phys. 148, 601-621 (1992)

20. Surgailis, D., Woyczynski, W.A.: Scaling limits of solutions of the Burgers' equation with singular Gaussian initial data, in Chaos Expansions, Multiple Wiener-Ito Integrals and Their Applications, C. Houdré and V. Pérez-Abreu, Eds, pp. 145-161, Boca Raton, Ann Arbor CRC Press (1994)

21. Woyczynski, W.A.: Stochastic Burgers' Flows. In: Nonlinear Waves and Weak Turbulence. Boston: Birkhäuser, pp. 279-311 (1993)

Communicated by Ya.G. Sinai 